\title{
KIDNEY CANCER
}

\section{Diabetes and low serum cholesterol are risk factors for RCC}

Dysregulated metabolism is emerging as an important contributor to renal cell carcinoma (RCC) pathogenesis, igniting interest in the associations between RCC and various metabolic factors. Two recently published studies contribute to our understanding of this disease, showing that diabetes mellitus and low serum cholesterol are associated with worse survival of patients with RCC.

The link between diabetes and RCC has been unclear for some time, with available evidence to support and dispute such an association. To overcome previous limitations of small sample sizes and confounding factors, Sarah Psutka and colleagues further investigated this relationship in a large matched-cohort study, derived from a series of 1,964 consecutive patients who underwent nephrectomy for RCC.

257 diabetic patients were matched 1:2 with 514 nondiabetic controls for a number of factors including sex, age, smoking status, performance status, severity of chronic kidney disease and type of surgery. However, they could not be matched for Charlson comorbidity index (CCI) or BMI owing to imbalance between groups. On multivariable analysis adjusting for CCI and BMI, patients with diabetes were at $55 \%$ and $32 \%$ increased risk of cancer-specific and all-cause mortality, respectively, than patients without diabetes. "The strength of this data set lies in its power and granularity, such that we were able to control for many possible observed confounders," says Psutka.

In the future, Psutka and her colleagues hope to unravel the mechanisms by which diabetes affects cancer-specific and overall survival in patients with RCC. "We are interested in understanding how this association might be modified with, for example, metformin, which has been demonstrated to have antineoplastic effects in other malignancies."

The prognostic role of serum cholesterol in patients with RCC is also uncertain, although some evidence exists to suggest that higher levels of cholesterol before nephrectomy are associated with significantly better survival and a greater response to systemic treatment with temsirolimus.

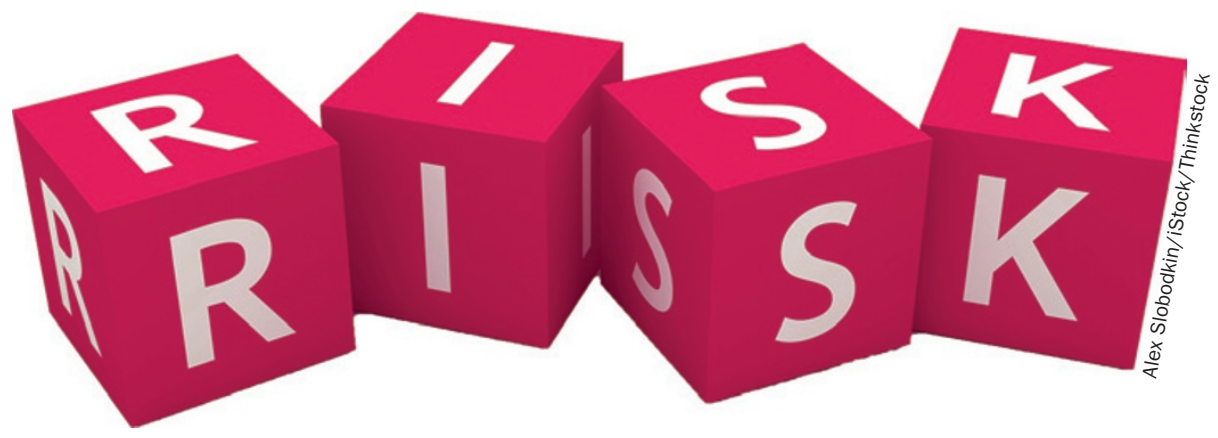

Given that most patients have their serum cholesterol routinely assessed when they are admitted to hospital, its use as a predictive factor is an attractive prospect. With this in mind, Tobias Klatte and colleagues at the Medical University of Vienna used the prospective kidney cancer database at their institution to evaluate the relationship between serum cholesterol before nephrectomy and survival in 867 patients with RCC.

The investigators found that for each $10 \mathrm{mg} / \mathrm{dl}$ increase in serum cholesterol, the risk of death from RCC decreased by $13 \%$. They also assessed the inclusion of serum cholesterol in a multivarable prognostic model. "For the first time, we were able to show that its use increases the discrimination of established prognostic factors and that cholesterol is an independent prognostic factor for patients who undergo surgery with curative intent," explains Klatte.

Klatte is keen to emphasize that their study is hypothesis-generating and their findings will, hopefully, be validated in a multicentre study that is currently underway. The biological underpinnings of the relationship between RCC and cholesterol requires further study.

\section{Sarah Payton}

Original articles Psutka, S. P. et al. Diabetes mellitus
is independently associated with an increased risk of
mortality among clear cell renal cell carcinoma patients.
J. Urol. doi:10.1016/j.juro.2014.06.014 | de Martino, M.
et al. Preoperative serum cholesterol is an independent
prognostic factor for patients with renal cell carcinoma
(RCC). BJU Int. doi:10.1111/bju.12767

